\section{A Systemic Review of Dupilumab Efficacy and Safety by Phenotypic Variations of Atopic Dermatitis}

\section{Piyu Parth Naik*}

Department of dermatology, Saudi German hospitals and clinics, Hessa Street 331 West, Al Barsha 3, Exit 36 Sheikh Zayed Road, Opposite of American School, Dubai, United Arab Emirates

\begin{abstract}
Background: The Atopic Dermatitis $(A D)$ is a chronic, pruritusinflammatory $\mathrm{TH} 2$ dominant skin disorder characterized by a reaction with complex immunopathogenesis. Dupilumab's effectiveness and safety profile by phenotypic variations of atopic dermatitis the treatment of atopic dermatitis has been investigated in a variety of large clinical trials in atopic dermatitis for more than 10 years. The goal of the current systematic review was to determine the effectiveness and protection of dupilumab through phenotypic differences in atopic dermatitis.

Methods: We systematically searched PubMed, MEDLINE, EMBASE and Google scholar databases to find appropriate articles published between last ten years related to dupilumab efficacy and safety by phenotypic variations of atopic dermatitis with the appropriate key terms (MeSH) including "dupilumab", "atopic dermatitis", "eczema", "phenotypic variations" "phenotypes" etc.

Results: Seven studies were included in the current review, which met the inclusion \& exclusion criteria of the current study. All the included studies in this review demonstrated that the dupilumab significantly treated the various phenotypes of $A D$, with the improvement of EASI and DLQI scores. The most commonly occurred side effect during the treatment of duplimab was allergic conjunctivitis followed by allergic rhinitis and asthma. Most of the included studies demonstrated that the dupilumab has been shown to rapidly improving signs and symptoms in the treatment of extreme AD.
\end{abstract}

*Corresponding author: Piyu Parth Naik, Department of dermatology, Saud German hospitals and clinics, Hessa Street 331 West, Al Barsha 3, Exit 36 Sheikh Zayed Road, Opposite of American School, Dubai, United Arab Emirates, Tel: +971 562173323; E-mail: drpiyu85@gmail.com

Citation: Naik PP (2021) A Systemic Review of Dupilumab Efficacy and Safety by Phenotypic Variations of Atopic Dermatitis. J Clin Dermatol Ther 7: 073.

Received: April 26, 2021; Accepted: April 30, 2021; Published: May 07, 2021

Copyright: (c) 2021 Naik PP. This is an open-access article distributed under the terms of the Creative Commons Attribution License, which permits unrestricted use, distribution, and reproduction in any medium, provided the original author and source are credited.

\begin{abstract}
Conclusion: Dupilumab was first prescribed for adults with atopic dermatitis and has been generally approved since 2018 for adult patients with moderate to extreme atopic dermatitis. The IL-4 and IL-13 cytokines in atopic dermatitis modulate the epidermal barrier and inhibit the production of antimicrobial peptides. Dupilumab's efficacy and safety profile in the treatment of atopic dermatitis has been investigated in a number of major clinical trials. This current systematic review provides an evidence-based report on dupilumab efficacy and safety by different phenotypic variations of atopic dermatitis, which will help to clinicians for better understanding of the mechanism of dupilumab against $A D$ phenotypes.
\end{abstract}

Keywords: Atopic dermatitis, Dupilumab; Endophenotypic variations; Interleukins, Pathogenesis

\section{Introduction}

Atopic Dermatitis (AD) is a chronic recurring inflammatory skin disease with an age-dependent anatomical localization characterized by pruritus and eczema. About $15-30 \%$ of children and $2-10 \%$ of adults are affected by $\mathrm{AD}$ [1-3]. It is increasingly recognized as a systemic disease, due primarily to transcriptomic [4], proteomic blood and skin [5]. AD diagnosis is based on the appearance of pruritis, eczema, normal anatomy, and chronic or recurring history. Other relevant characteristics include early life signs, atopy, increased overall and necessary $\operatorname{IgE}$ and xerosis [6]. The pathogenesis of genetic and environmental factors implicated in $\mathrm{AD}$ centers leads to the inflammatory process of the skin [7]. Up to $70 \%$ of patients have a history of atopic diseases in their families [8]. The high prevalence of AD since industrialization has been argued for urban lifestyles and other environmental causes, such as air pollution and tobacco smoke. There is an abundance of evidence that epigenetic modifications contribute to AD's pathogenesis in response to these environmental factors. DNA methylation, histone modification and the microRNA (miR) responsible for barrier activity and immune modulation are found in these epigenetic pathways [9]. AD's pathophysiology concerns both the skin membrane and immune abnormalities; certain clinical phenotypes and underlying endotypes are central to type 2 immune variance [10]. A broad variety of innate immune cells, including type 2 innate lymphoid cells, dendritic cells, eosinophils, mast cells and basophils, and adaptive immune cells such as T cells and $\mathrm{B}$ cells, have been identified as being involved in a complex immune network that leads to AD skin inflammation. It has been shown that the T-helper cells (Th2 cells) play a crucial causal role in $\mathrm{AD}$ [11]. The main source of interleukin (IL)-4, IL-5 prototypic (TH2) cytokines is these cells. AD patients have damaged the integrity of the skin and are more susceptible to allergens and pathogens that induce innate and adaptive immune responses [12].

Interleukin (IL)-4 and IL-13 play a crucial role in the pathogenesis of atopic dermatitis. Blocking both IL-4 and IL-13 signals to treat atopic dermatitis can be a strong and successful strategy. An IL-4R alpha inhibitor is a totally human monoclonal dupilumab antibody that 
ultimately blocks the signals IL-4 and IL-13. The treatment of atopic dermatitis has been investigated in a variety of large clinical trials in atopic dermatitis for more than ten years. Dupilumab inhibits several inflammatory pathways, including the activation of Th2 cells and developing various cytokines and chemokines. Dupilumab was first recommended for patient with atopic dermatitis and has been widely accepted since 2018 for adult patients with mild to serious atopic dermatitis $[13,14]$. Dupilumab injection is used for treating the effects of eczema (atopic dermatitis; a skin disease that causes the skin to be dry and itchy and sometimes develops red, scaly rashes). It is also used along with other medications to prevent wheezing, shortness of breath, coughing, and chest tightness due to certain asthma types [15]. Further advances in our knowledge of disease mechanisms that lead to the identification of particular phenotypes can enable us to further identify which of our patients would be the strongest candidates for specific treatments when we step towards a personalized approach to AD care [1]. Many double-blind, placebo-controlled trials have studied the therapeutic feasibility and safety of dupilumab in AD that observed substantial improvements in the Eczema Activity Severity Index (EASI) [16,17]. Also, several systematic reviews have been carried to find out the safety and efficacy of dupilumab in AD [18,19]. However, to our knowledge there are no systematic reviews were conducted on different clinical presentations of AD. Therefore, with many phenotypic differences of atopic dermatitis, the present research systematically reviews the effectiveness and protection of dupilumab.

\section{Materials and Methods}

\section{Study design}

The recommendations for Preferred Reporting Elements for Systematic Reviews and Meta-Analyses (PRISMA) have been followed in the present systematic analysis [20]. A systematic review comprises of the structured collection of evidence based articles followed by systematically discussing their findings based on the aim of the review.

\section{Search strategy}

An articles search was undertaken electronically using online databases such as Google scholar, MEDLINE, PubMed, Scopus and Cochrane databases with the appropriate key terms (MeSH). We were looking specifically for articles on dupilumab efficacy and safety by phenotypic variations of atopic dermatitis. The key words were changed according to each database's searching protocol including "Dupilumab", "Atopic dermatitis", "phenotypic variations of atopic dermatitis", "Dupilumab on dermatological disorders", "Atopic eczema", "Eczema". Bibliographic sources were also screened.

\section{Inclusion and exclusion criteria}

The articles published between the years 2016 and 2020 related to dupilumab efficacy and safety by phenotypic variations of $\mathrm{AD}$ was included in this review. The case studies, studies related to other than dermatological disorders, review and systematic review andthis research omitted papers written in a language other than English.

\section{Data extraction}

The initial literature search revealed 722 articles. Relevant articles were chosen for full-text screening after application of the eligibility criteria. The 'author and country', 'study design', 'sample size', 'study design', 'phenotypes', 'adverse effects', 'eczema area and severity index (EASI), Dermatology Life Quality Index (DLQI), and 'outcomes' were extracted from the selected articles.

\section{Results}

\section{Eligible studies}

The literature search yielded 722 articles from various databases including PubMed, Ovid, Science Direct and Google Scholar, of which 399 articles were excluded at the initial stage due to repetition and irrelevance. Out of 323, 151 articles were further excluded after analysis of the titles and abstracts at the first screening level. A total of 72 potential relevant articles were selected for full-text evaluations, of which 65 articles were further excluded as studies related to other diseases $(n=24)$, case reports $(n=24)$, review articles and systematic review and meta-analysis articles $(n=22)$. Finally, 7 studies meeting the inclusion criteria of the current systematic review as detailed in the PRISMA flow chart (Figure 1), were included in this review.

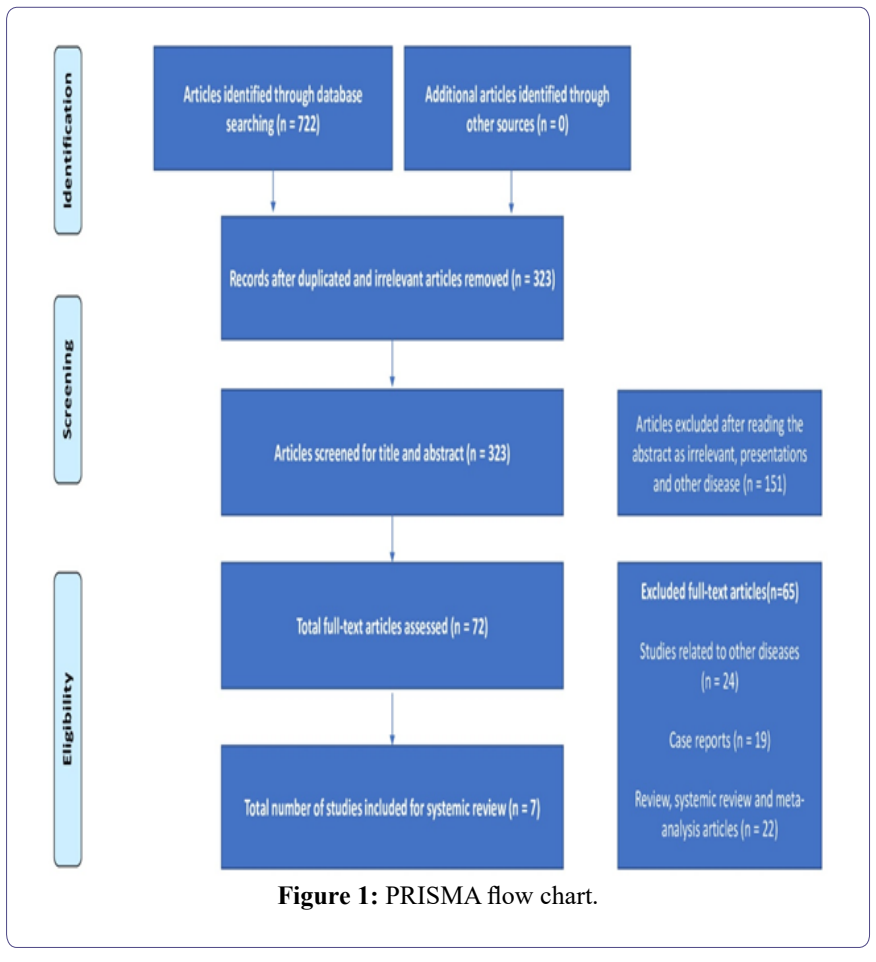

\section{Baseline characteristics study}

Among the included 7 articles, 6 studies retrospective design and one study randomized controlled trail [21-27]. Furthermore, 3 of the articles were published in the America [22,26,27], 2 articles were published in Italy [21,23], one article was published in Europe and one article was published in Australia [24,25]. The sample size ranged from 16 to 1491 patients, with a total of 2349 patients involved in the current systematic review and the age of the patients was 18 to 87 years. Treatment duration was ranging between 16 and 35.7 weeks.

\section{Clinical phenotypes of atopic dermatitis}

Among the studies involved, the influence of dupilumab was studied in seven separate phenotypes, including prurigo nodularis, 
generalized inflammatory eczema, classical, generalized lichenoid eczema, nummular eczema, erythroderma and pruritis.

\section{Prurigo nodularis}

The efficacy of dupilumab on prurigo nodularis were evaluated by 5 studies, of which one study evaluated that the generalized prurigo nodularis and one study evaluated chronic prurigo nodularis $[22,26]$.A significant improvement was observed in the Dermatology Life Quality Index (DLQI) and in the Eczema Area and Severity Index (EASI) after the treatment with dupilumab was reported by the retrospective study by Napolitano, et al. [22]. A retrospective study by Chiricozzi, et al. conducted among 27 patients with chronic prurigo nodularis reported that the dupilumab was effectively reduced the itch and also improving chronic prurigo nodularis skin lesions [26]. Similarly, a substantial change in prurigo phenotype was noted after 16 weeks of therapy with dupilumab $(\mathrm{p}<0.0001)$ and lasted until week 52 [21]. Also, several retrospective studies have reported the efficacy of dupilumab on prurigo nodularis $[24,25]$.

\section{Nummular eczema and generalized eczema}

The efficacy of dupilumab on nummular eczema was evaluated by Patruno, et al. among the 30 adult patients with nummular-like AD [23]. Dupilumab shows a substantial increase in the disease after 16 weeks of therapy, as shown by a decline in EASI and DLQI ratings. Similarly, Tavecchio, et al. reported a significant increase in nummular eczema [21], generalized inflammatory eczema and generalized phenotypic lichenoid pattern eczema after 16 weeks of therapy with dupilumab $(p<0.0001)$ and continued until week 52 . In addition, the authors stated that decreases in EASI were slower in nummular eczema phenotypes than in other phenotypes, suggesting that treatment should not be halted until an adequate therapeutic result has been obtained.

\section{Classic, erythroderma and pruritis}

Dupilumab shows clear and maintained effectiveness over a 76week treatment duration, decreased skin lesions and pruritus, and increased quality of life reported by Deleuran, et al. [27]. Tavecchio, et al. reported a possible change in $\mathrm{AD}$ classical and erythroderma phenotypes after 16 weeks of dupilumab therapy $(p<0.0001)$ and progressed until week 52 [21].

\section{Discontinuation due to adverse effects}

A study by Deleuran et al. reported that the due to adverse events $\mathrm{n}=27(1.8 \%)$ patients permanently discontinuation the treatment [27]. However, all the included studies demonstrated that most commonly occurred side effect during the treatment of duplimab was allergic conjunctivitis, except one study [21].Other frequently reported side effects were allergic rhinitis and asthma.

\section{Quality of life}

Most of the included studies demonstrated that the dupilumab has been shown to rapidly improving signs and symptoms in the treatment of extreme AD [21-23,26,27], including skin manifestations such as pruritus, lichenification, prurigo nodularis, excoriation, erythema and as well as sleep disruptions, edema/population and the overall quality of life of patients. Dupilumab is also well tolerated, with few adverse effects, including reactions at the injection site, conjunctivitis, nasopharyngitis, etc [25].

\section{Discussion}

Atopic Dermatitis (AD) is a chronic inflammatory and relapsing disease that can become chronic, characterized by eczematous spots, dry skin and extreme itching [18]. AD displays a heterogeneous phenotypes and highly complex pathophysiology. In adult-onset $\mathrm{AD}$ cases, in addition to the classical type, the clinical presentation is prurigo lesions, multiple lichenification areas, hand eczema or headand-neck dermatitis [28]. In the current study, we systematically review efficacy and safety of dupilumab with several phenotypic variations in atopic dermatitis. However, evidence found in the efficacy of dupilumab on different phenotypes of $\mathrm{AD}$ is limited, apart from case studies. All the included studies in this review demonstrated that the dupilumab significantly treated the various phenotypes of $\mathrm{AD}$, with the improvement of EASI and DLQI scores. Similarly, a review by Maloney, et al. reported that the dupilumab have shown efficacy for various conditions of AD [29].

Our findings showed that the most commonly occurred side effect during the treatment of duplimab was allergic conjunctivitis followed by allergic rhinitis and asthma.Previously,several studies reported that the dupilumab has a relatively safe side effect profile and the higher rates of conjunctivitis seen with dupilumab use thus far have only been found in $\mathrm{AD}$ patients [30,31]. In this review, the dupilumab has been shown to rapidly improving signs and symptoms in the treatment of extreme $\mathrm{AD}$ and improved the overall quality of life. Similarly, a report performed by Simpson et al. demonstrated substantial improvement in dupilumab in itchy [32], painful, irritated or stinging skin, feelings of shame and decreased sleep disruption. Although, it's potential for further dermatology applications in recalcitrant cases is a very interesting topic, although cost is a significant limiting factor. Finally, several more clinical trials and an improved knowledge of skin disease pathophysiology will help define the best possible dermatological applications of dupilumab [29].

The present review has some limitations. Firstly, eligible studies included in the present systematic review regarding the efficacy of dupilumabon various phenotypes of $\mathrm{AD}$ were performed with a wide range of treatment modalities and applications. Also, several side effects were also reported by the included studies. This variation points showed the fact that there has been a lack of standardized, universal and acceptable treatment for the AD. Secondly, case studies were not included in this review. Thirdly, the efficacy of dupilumab among various age groups, genders and ethnicities was not evaluated in this review as most of the studies did not report the efficacy of dupilumab on different endophenotypic variations. Despite these limitations, an evidence-based study on the effectiveness and protection of dupilumab on different $\mathrm{AD}$ phenotypes is provided in this current systematic review.

\section{Conclusion}

A fully human monoclonal antibody of dupilumab is an IL$4 \mathrm{R} \alpha$ inhibitor that blocks IL-4 and IL-13 signals subsequently. Dupilumab was first recommended for patient with $\mathrm{AD}$ and has been widely accepted since 2018 for adult patients who suffer from moderate to extreme AD. The IL-4 and IL-13 cytokines modulate the epidermal barrier in $\mathrm{AD}$ and inhibit antimicrobial peptide production. Effectiveness and safety profile of dupilumab in the treatment of allergic diseases has been investigated in a variety of large clinical trials in atopic dermatitis for more than 10 years. In long-term clinical 
trials, the safety profile of dupilumab has proved to be very favorable. In the current systematic review provided the efficacy of dupilumab on various clinical phenotypes of demonstrated by various studies. However, in order to evaluate its effectiveness and assess its best possible application, further studies will be required.

\section{References}

1. Asher MI, Montefort S, Björkstén B, Lai CKW, Strachan DP, et al. (2006) Worldwide time trends in the prevalence of symptoms of asthma, allergic rhinoconjunctivitis, and eczema in childhood: ISAAC Phases One and Three repeat multicountry cross-sectional surveys. Lancet 368: 733-743.

2. Hanifin JM, Reed ML, Eczema Prevalence and Impact Working Group (2007) A Population-Based Survey of Eczema Prevalence in the United States. Dermatitis 18: 82-91.

3. Silverberg JI, Simpson EL (2014) Associations of childhood eczema severity: a US population-based study. Dermatitis 25: 107-114.

4. Ungar B, Garcet S, Gonzalez J, Dhingra N, da Rosa JC, et al. (2017) An Integrated Model of Atopic Dermatitis Biomarkers Highlights the Systemic Nature of the Disease. J Invest Dermatol 137: 603-613.

5. Pavel AB, Zhou L, Diaz A, Ungar B, Dan J, et al. (2020) The proteomic skin profile of moderate-to-severe atopic dermatitis patients shows an inflammatory signature. J Am Acad Dermatol 82: 690-699.

6. Eichenfield LF, Ahluwalia J, Waldman A, Borok J, Udkoff J, et al. (2017) Current guidelines for the evaluation and management of atopic dermatitis: A comparison of the Joint Task Force Practice Parameter and American Academy of Dermatology Guidelines. J Allergy Clin Immunol 139: 49-57.

7. Boguniewicz M, Leung DYM (2011) Atopic dermatitis: a disease of altered skin barrier and immune dysregulation. Immunol Rev 242: 233-246.

8. Gupta J, Johansson E, Bernstein JA, Chakraborty R, Hershey GKK, et al. (2016) Resolving the etiology of atopic disorders by using genetic analysis of racial ancestry. J Allergy Clin Immunol 138: 676-699.

9. Liang Y, Chang C, Lu Q (2016) The Genetics and Epigenetics of Atopic Dermatitis-Filaggrin and Other Polymorphisms. Clin Rev Allergy Immunol 51: 315-328.

10. Boguniewicz M, Leung DYM (2015) Targeted therapy for allergic diseases: At the intersection of cutting-edge science and clinical practice. $\mathrm{J}$ Allergy Clin Immunol 135: 354-356.

11. Biedermann T, Skabytska Y, Kaesler S, Volz T (2015) Regulation of T Cell Immunity in Atopic Dermatitis by Microbes: The Yin and Yang of Cutaneous Inflammation. Front Immunol 6: 353.

12. Hammad H, Lambrecht BN (2015) Barrier Epithelial Cells and the Control of Type 2 Immunity. Immunity 43: 29-40.

13. Boguniewicz M (2017) Biologic Therapy for Atopic Dermatitis: Moving Beyond the Practice Parameter and Guidelines. J Allergy Clin Immunol Pract 5: 1477-1487.

14. Brunner PM, Guttman-Yassky E, Leung DYM (2017) The immunology of atopic dermatitis and its reversibility with broad-spectrum and targeted therapies. J Allergy Clin Immunol 139: 65-76.

15. Medlineplus (2015) Drugs, Herbs and Supplements.

16. Simpson EL, Thomas Bieber 1, Emma Guttman-Yassky 1, Lisa A Beck 1, Andrew Blauvelt, et al. (2016) Two Phase 3 Trials of Dupilumab versus Placebo in Atopic Dermatitis. N Engl J Med 375: 2335-2348.

17. M. de Bruin-Weller et al., "Dupilumab with concomitant topical corticosteroid treatment in adults with atopic dermatitis with an inadequate response or intolerance to ciclosporin A or when this treatment is medically inadvisable: a placebo-controlled, randomized phase III clinical trial (LIBERTY AD CAFÉ). Br J Dermatol 178: 1083-1101.
18. Agache I, Song Y, Posso M, Alonso-Coello P, Rocha C, et al., (2021) Efficacy and safety of dupilumab for moderate to severe atopic dermatitis: A systematic review for the EAACI biologicals guidelines. Allergy 76: 45-58.

19. Wang F-P, Tang X-J, Wei C-Q, Xu L-R, Mao H, et al. (2018) Dupilumab treatment in moderate-to-severe atopic dermatitis: A systematic review and meta-analysis. J Dermatol Sci 90: 190-198.

20. Liberati A, Altman DG, Tetzlaff J, Mulrow C, Gøtzsche PC, et al., "The PRISMA Statement for Reporting Systematic Reviews and Meta-Analyses of Studies That Evaluate Health Care Interventions: Explanation and Elaboration. PLoS Med 6: 100

21. Tavecchio S, Angileri L, Giuffrida FP, Germiniasi F, Marzano AV, et al. (2020) Efficacy of Dupilumab on Different Phenotypes of Atopic Dermatitis: One-Year Experience of 221 Patients. J Clin Med 9: 2684.

22. Napolitano M, Fabbrocini G, Scalvenzi M, Nisticò SP, Dastoli S, et al (2020) Effectiveness of Dupilumab for the Treatment of Generalized Prurigo Nodularis Phenotype of Adult Atopic Dermatitis," Dermatitis 31: 8184.

23. Patruno C, Stingeni L, Hansel K, Ferrucci SM, Tavecchio S, et al. (2020) Effectiveness of dupilumab for the treatment of nummular eczema phenotype of atopic dermatitis in adults. Dermatol Ther 33: 13290.

24. Calugareanu A, Jachiet M, Tauber M, Nosbaum A, Aubin F, et al. (2020) Effectiveness and safety of dupilumab for the treatment of prurigo nodularis in a French multicenter adult cohort of 16 patients. J Eur Acad Dermatology Venereol 34: 74-76.

25. Criado PR, Pincelli TP, Criado RFJ (2020) Dupilumab as a useful treatment option for prurigo nodularis in an elderly patient with atopic diathesis. Int J Dermatol 59: 358-361.

26. Chiricozzi A, Maurelli M, Gori N, Argenziano G, De Simone C, et al (2020) Dupilumab improves clinical manifestations, symptoms, and quality of life in adult patients with chronic nodular prurigo," J. Am. Acad. Dermatol 83: 39-45.

27. Deleuran M, Thaçi D, Beck LA, de Bruin-Weller M, Blauvelt A, et al. (2020) Dupilumab shows long-term safety and efficacy in patients with moderate to severe atopic dermatitis enrolled in a phase 3 open-label extension study. J Am Acad Dermatol 82: 377-388.

28. Patruno C, Fabbrocini G, Napolitano M (2020) Clinical phenotypes of atopic dermatitis of the adult. G Ital Dermatol Venereol.

29. Maloney NJ, Tegtmeyer K, Zhao J, Worswick S (2019) Dupilumab in Dermatology: Potential for Uses Beyond Atopic Dermatitis. J Drugs Dermatol 18: 1545961619P1053X.

30. Bachert C, Mannent L, Naclerio RM, Mullol J, Ferguson BJ, et al. (2016) Effect of Subcutaneous Dupilumab on Nasal Polyp Burden in Patients With Chronic Sinusitis and Nasal Polyposis: A Randomized Clinical Trial. JAMA 315: 469-479.

31. Wenzel S, Castro M, Corren J, Maspero J, Wang L, et al. (2016) Dupilumab efficacy and safety in adults with uncontrolled persistent asthma despite use of medium-to-high-dose inhaled corticosteroids plus a long-acting $\beta 2$ agonist: A randomised double-blind placebo-controlled pivotal phase $2 \mathrm{~b}$ dose-ranging trial. Lancet 388: 31-44.

32. Simpson E, Beck L, Gadkari A, Eckert L, Reaney M, et al. (2017) Defining a responder on the Peak Pruritus Numerical Rating Scale (NRS) in patients with moderate-to-severe atopic dermatitis: Detailed analysis from randomized trials of dupilumab. J. Am Acad Dermatol 97: 1045-1046. 


\section{di \\ нетан}

Advances In Industrial Biotechnology | ISSN: 2639-5665

Advances In Microbiology Research | ISSN: 2689-694X

Archives Of Surgery And Surgical Education | ISSN: 2689-3126

Archives Of Urology

Archives Of Zoological Studies | ISSN: 2640-7779

Current Trends Medical And Biological Engineering

International Journal Of Case Reports And Therapeutic Studies | ISSN: 2689-310X

Journal Of Addiction \& Addictive Disorders | ISSN: 2578-7276

Journal Of Agronomy \& Agricultural Science | ISSN: 2689-8292

Journal Of AIDS Clinical Research \& STDs | ISSN: 2572-7370

Journal Of Alcoholism Drug Abuse \& Substance Dependence | ISSN: 2572-9594

Journal Of Allergy Disorders \& Therapy | ISSN: 2470-749X

Journal Of Alternative Complementary \& Integrative Medicine | ISSN: 2470-7562

Journal Of Alzheimers \& Neurodegenerative Diseases | ISSN: 2572-9608

Journal Of Anesthesia \& Clinical Care | ISSN: 2378-8879

Journal Of Angiology \& Vascular Surgery | ISSN: 2572-7397

Journal Of Animal Research \& Veterinary Science | ISSN: 2639-3751

Journal Of Aquaculture \& Fisheries | ISSN: 2576-5523

Journal Of Atmospheric \& Earth Sciences | ISSN: 2689-8780

Journal Of Biotech Research \& Biochemistry

Journal Of Brain \& Neuroscience Research

Journal Of Cancer Biology \& Treatment | ISSN: 2470-7546

Journal Of Cardiology Study \& Research | ISSN: 2640-768X

Journal Of Cell Biology \& Cell Metabolism | ISSN: 2381-1943

Journal Of Clinical Dermatology \& Therapy | ISSN: 2378-8771

Journal Of Clinical Immunology \& Immunotherapy | ISSN: 2378-8844

Journal Of Clinical Studies \& Medical Case Reports | ISSN: 2378-8801

Journal Of Community Medicine \& Public Health Care | ISSN: 2381-1978

Journal Of Cytology \& Tissue Biology | ISSN: 2378-9107

Journal Of Dairy Research \& Technology | ISSN: 2688-9315

Journal Of Dentistry Oral Health \& Cosmesis | ISSN: 2473-6783

Journal Of Diabetes \& Metabolic Disorders | ISSN: 2381-201X

Journal Of Emergency Medicine Trauma \& Surgical Care | ISSN: 2378-8798

Journal Of Environmental Science Current Research | ISSN: 2643-5020

Journal Of Food Science \& Nutrition | ISSN: 2470-1076

Journal Of Forensic Legal \& Investigative Sciences | ISSN: 2473-733X

Journal Of Gastroenterology \& Hepatology Research | ISSN: 2574-2566
Journal Of Genetics \& Genomic Sciences | ISSN: 2574-2485

Journal Of Gerontology \& Geriatric Medicine | ISSN: 2381-8662

Journal Of Hematology Blood Transfusion \& Disorders | ISSN: 2572-2999

Journal Of Hospice \& Palliative Medical Care

Journal Of Human Endocrinology | ISSN: 2572-9640

Journal Of Infectious \& Non Infectious Diseases | ISSN: 2381-8654

Journal Of Internal Medicine \& Primary Healthcare | ISSN: 2574-2493

Journal Of Light \& Laser Current Trends

Journal Of Medicine Study \& Research | ISSN: 2639-5657

Journal Of Modern Chemical Sciences

Journal Of Nanotechnology Nanomedicine \& Nanobiotechnology | ISSN: 2381-2044

Journal Of Neonatology \& Clinical Pediatrics | ISSN: 2378-878X

Journal Of Nephrology \& Renal Therapy | ISSN: 2473-7313

Journal Of Non Invasive Vascular Investigation | ISSN: 2572-7400

Journal Of Nuclear Medicine Radiology \& Radiation Therapy | ISSN: 2572-7419

Journal Of Obesity \& Weight Loss | ISSN: 2473-7372

Journal Of Ophthalmology \& Clinical Research | ISSN: 2378-8887

Journal Of Orthopedic Research \& Physiotherapy | ISSN: 2381-2052

Journal Of Otolaryngology Head \& Neck Surgery | ISSN: 2573-010X

Journal Of Pathology Clinical \& Medical Research

Journal Of Pharmacology Pharmaceutics \& Pharmacovigilance | ISSN: 2639-5649

Journal Of Physical Medicine Rehabilitation \& Disabilities | ISSN: 2381-8670

Journal Of Plant Science Current Research | ISSN: 2639-3743

Journal Of Practical \& Professional Nursing | ISSN: 2639-5681

Journal Of Protein Research \& Bioinformatics

Journal Of Psychiatry Depression \& Anxiety | ISSN: 2573-0150

Journal Of Pulmonary Medicine \& Respiratory Research | ISSN: 2573-0177

Journal Of Reproductive Medicine Gynaecology \& Obstetrics | ISSN: 2574-2574

Journal Of Stem Cells Research Development \& Therapy | ISSN: 2381-2060

Journal Of Surgery Current Trends \& Innovations | ISSN: 2578-7284

Journal Of Toxicology Current Research | ISSN: 2639-3735

Journal Of Translational Science And Research

Journal Of Vaccines Research \& Vaccination | ISSN: 2573-0193

Journal Of Virology \& Antivirals

Sports Medicine And Injury Care Journal | ISSN: 2689-8829

Trends In Anatomy \& Physiology | ISSN: 2640-7752

Submit Your Manuscript: https://www.heraldopenaccess.us/submit-manuscript 